\title{
Membranas de PVA e Sílica para Aplicação em Célula a Combustível de Alimentação Direta de Álcool
}

\author{
Liz Contino Vianna de Aguiar, Ailton de Souza Gomes \\ Instituto de Macromoléculas Professora Eloisa Mano - IMA, Universidade Federal do \\ Rio de Janeiro - UFRJ \\ Florêncio Gomes de Ramos Filho \\ Universidade Estadual da Zona Oeste - UEZO
}

\begin{abstract}
Resumo: Membranas de PVA/sílica foram sintetizadas através do processo sol-gel em condições ácidas. Tetraetilortossilicato (TEOS) foi utilizado como precursor em concentrações de 5 a $30 \%$ em dois grupos distintos. Um grupo foi utilizado como controle contendo apenas PVA/TEOS. A inserção dos grupamentos necessários para a condutividade ocorreu através da introdução de $12 \%$ em massa de heteropoliácido fosfotúngstico hidratado (HPW) em relação à massa de TEOS, formando o segundo grupo. As avaliações quanto ao grau de inchamento, permeabilidade ao etanol e condutividade protônica demonstraram que a membrana contendo 30\% em massa de TEOS apresenta os melhores resultados entre as membranas produzidas. Os melhores resultados foram $0,28 \mathrm{mS} / \mathrm{cm}$ de condutividade protônica e fluxo de $1,5 \mathrm{~kg} / \mathrm{m}^{2}$.h de solução de etanol. Nessas condições, considera-se a reticulação das membranas de $\mathrm{PVA} / \mathrm{SiO}_{2}$ com o precursor TEOS uma boa alternativa para a reticulação de membranas de PVA visto que as membranas mostraram-se mais seletivas ao fluxo de solução de etanol. Entretanto, para aplicação como eletrólito de célula a combustível, ainda é necessário investigar outras formas de aumentar a condutividade sem prejudicar a estabilidade dimensional.
\end{abstract}

Palavras-chave: $P V A$, célula a combustivel, $H P W$, sílica, membranas condutoras de prótons.

\section{Membranes of PVA and Silica for Application in Direct Alcohol Fuel Cell}

\begin{abstract}
PVA/silica membranes were synthesized by the sol-gel process under acidic conditions. Tetraethoxysilane (TEOS) was used as precursor with concentration between 5 and 30\% in two distinct systems. One system was used as control, which contained only PVA / TEOS. In the second system, groups necessary for conductivity were introduced with $12 \%$ by weight of hydrated phosphotungstic heteropolyacid (HPW) in relation to the mass of TEOS. The evaluations of the degree of swelling, permeability for ethanol and proton conductivity have shown that the membrane containing 30 $\mathrm{wt} \%$ TEOS gives the best results among the membranes produced. The best results achieved in this study were $0.28 \mathrm{mS} /$ $\mathrm{cm}$ of proton conductivity and $1.5 \mathrm{~kg} / \mathrm{m}^{2}$.h flux of ethanol solution. Thus, under these conditions, crosslinked membranes with PVA/SiO2 and TEOS precursor are good alternative for crosslinking PVA membranes since they were more selective to the flow of ethanol solution. However, for applications such as in electrolyte fuel cells, it is still necessary to investigate other ways to increase conductivity without affecting the dimensional stability.
\end{abstract}

Keywords: PVA, fuel cell, HPW, silica, proton conducting membranes.

\section{Introdução}

Célula a combustível (FC) que utiliza membrana polimérica condutora de prótons tem despertado bastante interesse ao longo dos anos devido ao seu baixo potencial poluidor e à larga gama de aplicação como em veículos e dispositivos portáteis ${ }^{[1-3]}$.

Tradicionalmente o Nafion, copolímero sulfonado perfluorado, é o mais utilizado em FC, entretanto apresenta o inconveniente do fenômeno de metanol crossover. Além desse problema técnico, o fato de ser um polímero caro motiva a busca por membranas alternativas ${ }^{[3-5]}$.

O principal interesse em utilizar o PVA como matriz polimérica para utilização em células a combustível alimentadas diretamente por álcool (DAFC) encontra-se no fato dele ter baixo custo e apresentar excelente propriedade de barreira à permeação de moléculas de alcoóis ${ }^{[6-8]}$.

Dois fatores podem comprometer a utilização de PVA em DAFC que são o elevado grau de inchamento e a ausência de grupos condutores de prótons em sua estrutura. Sistemas de materiais híbridos, composto por uma fase orgânica e outra inorgânica, são promissores em muitos campos de aplicação tecnológica, devido ao sinergismo apresentado por esses compostos ${ }^{[9-13]}$.

O processo sol gel baseia-se na polimerização de precursores moleculares, tais como alcóxidos metálicos, e inclui como etapas um estado de solução coloidal (sol), a passagem para um estado de gel e a remoção do solvente. O termo sol é empregado para definir uma dispersão de

Autor para correspondência: Liz Contino Vianna de Aguiar, Instituto de Macromoléculas Professora Eloisa Mano - IMA, Centro de Tecnologia, Universidade Federal do Rio de Janeiro - UFRJ, Bloco J, Cidade Universitária, Ilha do Fundão, CP 68525, CEP 21945-970, Rio de Janeiro, RJ, Brasil, e-mail: lizcontino@ima.ufrj.br 
partículas coloidais (dimensões entre 1 a 100 nm) estável em um fluido, enquanto que o termo gel refere-se ao sistema formado pela estrutura rígida de partículas coloidais (gel coloidal) ou de cadeias poliméricas (gel polimérico) que imobiliza a fase líquida nos seus interstícios ${ }^{[14,15]}$.

As propriedades físicas e químicas dos materiais resultantes dependem em grande parte das condições de reação. Logo, o catalisador utilizado é que determinará a forma de crescimento da espécie condensada ${ }^{[12,14,16,17]}$.

No caso dos alcoóxidos de silício, o catalisador ácido mais utilizado na sua hidrólise é o $\mathrm{HCl}$, e a catálise ácida resulta polímeros de cadeia maior que se emaranham, formando géis poliméricos. Em condições básicas, a reação de hidrólise/condensação é extremamente rápida formando partículas esféricas de sílica, podendo gerar aglomerados das partículas inorgânicas ${ }^{[12,14,15,17]}$.

O PVA com elevado grau de hidrólise possui um elevado número de grupos hidroxilas. Por meio desses grupos é esperado que ocorram fortes interações como ligação de hidrogênio e ligações secundárias, covalentes, entre a hidroxila da cadeia do PVA e o grupo silanol que foi gerado no processo sol-gel com catálise ácida. Essas interações garantem a homogeneidade das membranas híbridas $^{[7,16]}$.

Frente às referências encontradas na literatura e objetivando uma membrana com boa condutividade protônica e redução do fluxo permeado de álcool, o presente estudo visa desenvolver membranas de $\mathrm{PVA} / \mathrm{SiO}_{2}$ através do processo sol-gel e avaliar as suas propriedades quanto ao grau de inchamento, permeabilidade em água e em etanol, condutividade protônica e estabilidade térmica.

\section{Experimental}

\section{Materiais}

Os reagentes utilizados são de seguintes proveniências. Poli(álcool vinílico) (procedência: Sigma - Aldrich, 99\% hidrolisado, massa molecular média 85.000-124.000, usado como recebido). Tetraetilortossilicato $\left(\mathrm{C}_{8} \mathrm{H}_{20} \mathrm{O}_{4} \mathrm{Si}\right)$ (TEOS) (procedência: Fluka, grau de pureza P.A., usado como recebido). Heteropoliácido fosforotungstico hidratado $\left(\mathrm{H}_{3} \mathrm{PW}_{12} \mathrm{O}_{40} \cdot \mathrm{xH}_{2} \mathrm{O}\right)$ (HPW) (procedência: Fluka, grau de pureza P.A).

\section{Métodos}

\section{Membranas híbridas}

7\% em massa de PVA foram dissolvidos em água deionizada a $100{ }^{\circ} \mathrm{C}$ por aproximadamente $30 \mathrm{~min}$. Em seguida, foi adicionado o precursor TEOS em quantidades variadas. O segundo grupo foi composto por, além do PVA e do precursor, $12 \%$ em massa de HPW em relação à massa de TEOS.

As soluções foram vazadas em placas de petri de plástico, previamente aquecidas a $\left(50^{\circ} \mathrm{C}\right)$, e permaneceram por $24 \mathrm{~h}$ para evaporação da água e formação da membrana. A secagem terminou com o aquecimento na estufa a vácuo a $100{ }^{\circ} \mathrm{C}$ por $24 \mathrm{~h}$.

A relação de membranas preparadas para o desenvolvimento deste trabalho, bem como a quantidade de precursor inorgânico presente em cada uma e a nomenclatura utilizada durante o trabalho está listada na Tabela 1.

\section{Caracterizações}

Análise de espectometria no infravermelho

A Espectometria no infravermelho com transformada de Fourrier (FTIR) foi realizada no espectrômetro Varian. As amostras foram analisadas com o acessório de Reflectância Total Atenuada (ATR). A faixa do espectro analisada foi de $4000 \mathrm{a} 500 \mathrm{~cm}^{-1}$. Esta técnica foi utilizada para a verificação da presença dos grupamentos funcionais dos componentes individuais nas membranas híbridas e, com isso mostrar a ocorrência de reação de reticulação entre a matriz de PVA e a sílica.

\section{Sorção em água e em solução de etanol}

As membranas foram cortadas em discos do mesmo tamanho e então foram secas e pesadas para obter o peso seco (PS). Em seguida, cada disco de membrana foi imerso em água deionizada e em solução de etanol $20 \%$, separadamente. $\mathrm{O}$ teste foi feito a $30{ }^{\circ} \mathrm{C}$ e a $60{ }^{\circ} \mathrm{C}$. Após o período de $26 \mathrm{~h}$ as amostras foram pesadas novamente para ter o peso úmido (PU) e o teor de água e de etanol sorvido (TS) pela membrana foi calculado pela Equação 1:

$$
\mathrm{TS}=(\mathrm{PU}-\mathrm{PS}) / \mathrm{PS} \times 100 \%
$$

\section{Pervaporação em água e em solução de etanol}

O ensaio de pervaporação consiste em forçar um fluido a permear pela membrana em função da aplicação de vácuo em uma das suas faces, enquanto a outra fica em contato com a quantidade a ser pervaporada.

Para a pervaporação utilizou-se um equipamento montado no laboratório. Nele, a membrana, cortada em disco, foi colocada dentro da célula onde a face superior é constantemente alimentada por uma solução aquosa de etanol ou água deionizada, e a face inferior é submetida a vácuo constante para que ocorra a permeação. $\mathrm{O}$ vapor permeado pela membrana é condensado e recolhido em traps imersos em nitrogênio líquido.

O ensaio foi realizado com o fluxo de água e também com uma solução aquosa de etanol $20 \%$ em massa a temperatura de $50^{\circ} \mathrm{C}$. O material pervaporado foi recolhido três vezes com intervalo de $1 \mathrm{~h}$ entre coletas. A quantidade de material pervaporado foi calculada através da diferença de massa do trap seco (antes de começar o teste) e do trap com o material pervaporado. Foi calculada a média das três medidas.

\section{Condutividade protônica}

Nesse trabalho foi utilizada a espectroscopia de impedância.

Tabela 1. Composição e nomenclatura das membranas.

\begin{tabular}{cl}
\hline Nomenclatura & \multicolumn{1}{c}{ Composição (\% em massa) } \\
\hline PVA & $100 \%$ PVA \\
TH75 & $64,10 \% \mathrm{PVA}+32,05 \% \mathrm{TEOS}+3,93 \% \mathrm{HPW}$ \\
TH19 & $87,56 \% \mathrm{PVA}+11,1 \% \mathrm{TEOS}+1,34 \% \mathrm{HPW}$ \\
TH9 & $93,70 \% \mathrm{PVA}+5,62 \% \mathrm{TEOS}+0,68 \% \mathrm{HPW}$ \\
\hline
\end{tabular}


As membranas foram previamente condicionadas em água deionizada a $60^{\circ} \mathrm{C}$ por $1 \mathrm{~h}$ e em seguida foram dispostas entre dois eletrodos de grafite. O conjunto membrana/eletrodo foi colocado dentro de uma célula de aço inoxidável

A célula foi colocada na estufa e as análises foram realizadas nas temperaturas de 30 e $60{ }^{\circ} \mathrm{C}$ utilizando o equipamento Autolab PGSTAT-30. A condutividade protônica pode ser calculada através da Equação 2.

$$
\sigma=1 / \mathrm{RS}
$$

onde, $\sigma$ é a condutividade de prótons, lé a distância entre os eletrodos (espessura entre as membranas), R é a impedância da membrana e S é a área de condução da membrana.

\section{Resultados e Discussões}

\section{Espectrometria de infravermelho com transformada de Fourier (FTIR)}

A Figura 1 mostra os resultados da análise de FTIR das membranas contendo o precursor TEOS.

O HPW hidratado tem bandas características na região de $1074 \mathrm{~cm}^{-1}$, referentes à dupla ligação entre o fósforo e o oxigênio, $974 \mathrm{~cm}^{-1}$, correspondente à ligação $\mathrm{W}=\mathrm{O}$ e a região de 895 e $769 \mathrm{~cm}^{-1}$, correspondente à ligação $\mathrm{W}-\mathrm{O}-\mathrm{W}$. Porém, não foi possível verificar os picos característicos do HPW na membrana modificada com o mesmo, Figura $1^{[18]}$.

Através da análise da Figura 1, foi possível observar a redução na faixa entre $3500-3000 \mathrm{~cm}^{-1}$ da hidroxila. Essa redução foi mais acentuada para a membrana TH75 do que para a membrana T75. Tal fato ocorreu possivelmente devido à reação entre o TEOS e a hidroxila do PVA que foi mais pronunciada quando o HPW foi incorporado.

A maior quantidade de HPW utilizada no trabalho foi $3,93 \%$, o que provavelmente tenha sido uma quantidade pequena e as bandas características do HPW ficaram suprimidas pelas bandas do PVA.

\section{Sorção em água e em solução de etanol}

A Tabela 2 apresenta o resultado do ensaio de sorção de água a $30^{\circ} \mathrm{C}$ e a $60^{\circ} \mathrm{C}$. A partir da análise dos valores da tabela pode-se verificar que as membranas obtidas com

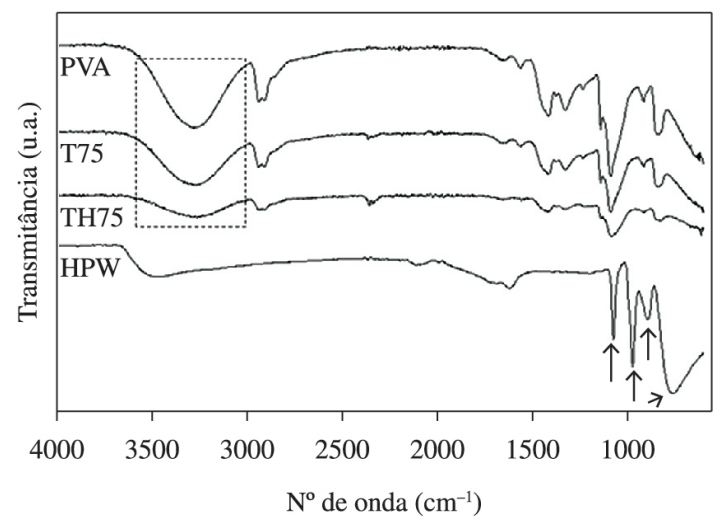

Figura 1. Análise de FTIR das membranas contendo TEOS. incorporação de sílica absorveu menor quantidade de água em relação à membrana de PVA pura. Esse comportamento pode ser atribuído à formação de ligações cruzadas durante a reticulação que restringi movimento das cadeias do polímero, reduzindo o volume disponível para a entrada das moléculas de água. Assim, as membranas híbridas apresentam melhor estabilidade dimensional ${ }^{[6]}$.

De forma geral, com exceção da amostra T75, as membranas apresentaram redução da absorção de água ao aumentar a temperatura para $60^{\circ} \mathrm{C}$. Possivelmente, a reação de reticulação continuou durante o teste de absorção quando a temperatura foi elevada para $60^{\circ} \mathrm{C}$. Para a membrana com maior teor de sílica (T75) isso não ocorreu a possivelmente devido à necessidade de temperatura mais elevada, acima de $60{ }^{\circ} \mathrm{C}$, para completar o seu processo de reticulação.

A Tabela 3 mostra o resultado os valores de sorção em água para as membranas com HPW. Analisando o resultado obtido a $30^{\circ} \mathrm{C}$, verifica-se que essas membranas absorvem mais água em relação às membranas híbridas obtidas sem HPW. Tal comportamento pode ser explicado pelo fato de HPW ser altamente higroscópico ${ }^{[19]}$.

Ao aumentar a temperatura para $60^{\circ} \mathrm{C}$, as membranas híbridas com HPW apresentam maior redução de absorção de água. Tal fato pode ser explicado pela ação catalítica do HPW na reação de reticulação do sistema, deixando a rede de cadeias mais densa com a possível formação de uma rede tridimensional entre a matriz de PVA, a sílica e o HPW ${ }^{[18-22]}$.

Ao se utilizar a membrana como eletrólito em células de alimentação direta de álcool, a sorção de etanol também é uma preocupação, visto que, qualquer alteração no caminho que o combustível deve seguir compromete o desempenho do sistema como um todo. Os resultados da sorção de solução de etanol $20 \%$ das membranas que contem o precursor TEOS são apresentados na Tabela 4.

Tabela 2. Sorção em água (\%) das membranas contendo o precursor TEOS em função da temperatura.

\begin{tabular}{ccc}
\hline \multirow{2}{*}{ Amostras } & \multicolumn{2}{c}{ TS } \\
\cline { 2 - 3 } & $\mathbf{3 0}^{\circ} \mathbf{C}$ & $\mathbf{6 0}^{\circ} \mathbf{C}$ \\
\hline PVA & 111,79 & 31,33 \\
T9 & 77,80 & 35,70 \\
T19 & 69,10 & 25,55 \\
T75 & 39,00 & 65,80 \\
\hline
\end{tabular}

Tabela 3. Sorção em água (\%) das membranas híbridas contendo HPW em função da temperatura.

\begin{tabular}{ccc}
\hline \multirow{2}{*}{ Amostras } & \multicolumn{2}{c}{ TS } \\
\cline { 2 - 3 } & $\mathbf{3 0}^{\circ} \mathbf{C}$ & $\mathbf{6 0}^{\circ} \mathbf{C}$ \\
\hline TH9 & 173,60 & 17,51 \\
TH19 & 137,33 & 16,51 \\
TH75 & 154,88 & 23,05 \\
\hline
\end{tabular}

Tabela 4. Sorção em solução de EtOh $20 \%$ (\%) das membranas com TEOS em função da temperatura.

\begin{tabular}{ccc}
\hline \multirow{2}{*}{ Amostra } & \multicolumn{2}{c}{ TS } \\
\cline { 2 - 3 } & $\mathbf{3 0}^{\circ} \mathbf{C}$ & $\mathbf{6 0}^{\circ} \mathbf{C}$ \\
\hline PVA & 226,27 & - \\
T9 & 154,42 & 311,63 \\
T19 & 103,88 & 268,85 \\
T75 & 70,51 & 134,63 \\
\hline
\end{tabular}


Em geral, as membranas híbridas obtidas com o precursor TEOS apresentaram menor sorção que a membrana de PVA pura, devido a reticulação das cadeias com $\mathrm{SiO}_{2}$.

Na sorção da solução de etanol $20 \%$ a $30{ }^{\circ} \mathrm{C}$ e a $60{ }^{\circ} \mathrm{C}$ das membranas do precursor TEOS, conforme é aumentada a quantidade de precursor no sistema, a porcentagem de sorção da solução de etanol diminui devido ao aumento da reticulação das cadeias do polímero com as partículas de sílica.

Os resultados da sorção em solução de etanol das membranas contendo TEOS/HPW são mostrados na Tabela 5 .

A modificação das membranas de TEOS com HPW reduziu o volume de sorção de etanol tanto na temperatura de $30{ }^{\circ} \mathrm{C}$ como na temperatura de $60{ }^{\circ} \mathrm{C}$, isto porque o efeito da reticulação com $\mathrm{SiO}_{2}$ prevaleceu sobre o efeito higroscópico do HPW. Além disso, as membranas de TEOS/ HPW apresentaram menor grau de sorção da solução de etanol quando comparadas com as membranas de PVA/ TEOS o que pode ser explicado pela melhor reticulação das cadeias já que o HPW é um ácido forte que catalisa a hidrólise do TEOS.

\section{Pervaporação em água e em solução de etanol}

Os resultados do teste de pervaporação (Figure 2) mostram a seletividade das membranas através da

Tabela 5. Sorção em solução de EtOH das membranas contendo TEOS/HPW em função da temperatura.

\begin{tabular}{ccc}
\hline $\begin{array}{c}\text { Amostra } \\
\text { modificada }\end{array}$ & \multicolumn{2}{c}{ TS } \\
\cline { 2 - 3 } & $\mathbf{3 0}^{\circ} \mathbf{C}$ & $\mathbf{6 0}^{\circ} \mathbf{C}$ \\
\hline TH9 & 173,61 & 17,51 \\
TH19 & 137,34 & 16,51 \\
TH75 & 153,43 & 23,05 \\
\hline
\end{tabular}

comparação entre a quantidade de fluxo permeado de água e de solução de etanol.

A seletividade das membranas híbridas obtidas com TEOS/HPW avaliada a $30^{\circ} \mathrm{C}$ é mostrada na Figura $2 \mathrm{a}$. De forma geral, a introdução das partículas inorgânicas reduz o volume do fluxo pervaporado da solução de etanol. Pode-se observar que as membranas híbridas de TEOS com HPW são mais seletivas a passagem da solução de etanol que a membrana de PVA pura, visto que em todas elas o volume pervaporado de água foi maior que o de solução de etanol.

$\mathrm{Na}$ Figura 2b, os valores de fluxo pervaporado da solução de etanol a $50{ }^{\circ} \mathrm{C}$ estão em uma faixa menor (mínimo de 1,39 e máximo de 3,03) do que quando analisados a $30{ }^{\circ} \mathrm{C}$ (mínimo de 2,97 e máximo de 4,09). Apesar disto, nessa temperatura as membranas PVA/ TEOS/HPW são consideradas menos seletivas, visto que a diferença entre os valores do fluxo pervaporado de água e solução de etanol são menores.

\section{Condutividade protônica}

A condutividade protônica das membranas sintetizadas neste trabalho foi apresentada na Tabela 6 .

O HPW é conhecido na literatura como um material condutor utilizado para aumentar a condutividade protônica das membranas utilizadas em célula a combustível. Este material tem forte tendência a migrar para fora da membrana e por isso precisa de um agente que o fixe na estrutura do polímero, por exemplo, sílica ${ }^{[22,23]}$.

$\mathrm{Li}, \mathrm{Xu}$ e Wang ${ }^{[24]}$ sintetizaram membranas condutoras de PVA/PWA em dimetilsulfóxido, alcançando valores de condutividade aproximados a $6 \mathrm{mS} / \mathrm{cm}$. Entretanto, para atingir tal resultado, foi utilizado até $60 \%$ de heteropoliácido perdendo a característica orgânica da membrana.

Apesar das membranas sintetizadas no trabalho passarem por um tratamento prévio para a realização das

Tabela 6. Condutividade das membranas sintetizadas em $(\mathrm{mS} / \mathrm{cm})$ em função da temperatura.

\begin{tabular}{|c|c|c|c|c|c|}
\hline \multirow{2}{*}{ Amostras sem HPW } & \multicolumn{2}{|c|}{ Condutividade } & \multirow{2}{*}{ Amostras com HPW } & \multicolumn{2}{|c|}{\begin{tabular}{|l} 
Condutividade \\
\end{tabular}} \\
\hline & $30{ }^{\circ} \mathrm{C}$ & $60^{\circ} \mathrm{C}$ & & $30^{\circ} \mathrm{C}$ & $60^{\circ} \mathrm{C}$ \\
\hline T9 & 0,05 & 0,01 & TH9 & 0,08 & 0,09 \\
\hline $\mathrm{T} 19$ & 0,02 & 0,07 & TH19 & 0,03 & 0,08 \\
\hline $\mathrm{T} 75$ & 0,06 & 0,07 & TH75 & 0,11 & 0,28 \\
\hline
\end{tabular}

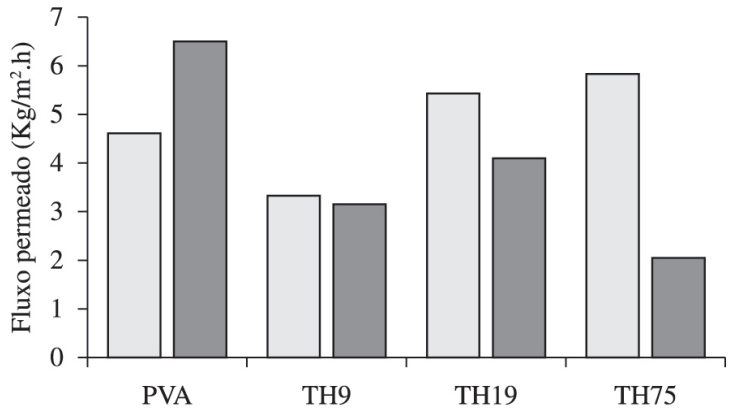

$\square$ Fluxo de água $\square$ Fluxo de sol. de EtOH (20\%)

(a)

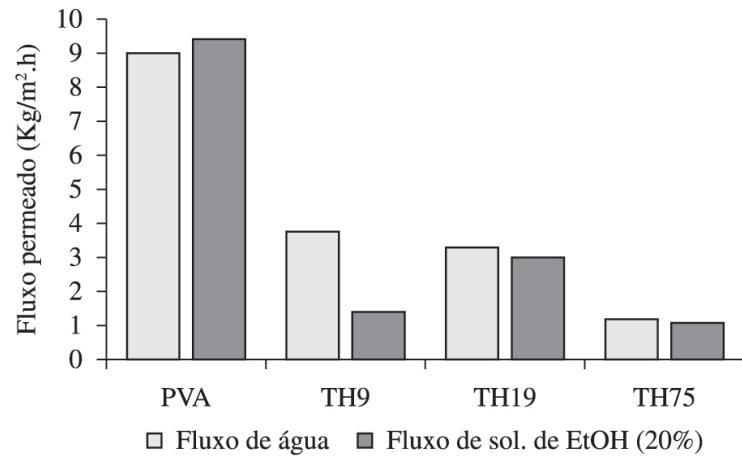

(b)

Figura 2. a e b fluxo permeado das membranas que contem TEOS/HPW a $30{ }^{\circ} \mathrm{C}$ e a $50{ }^{\circ} \mathrm{C}$ respectivamente. 
medidas, assim como esperado, a introdução do HPW nas cadeias do PVA aumentou a condutividade, mostrando que alguma quantidade de HPW permaneceu na membrana, devido a reticulação alcançada com as partículas de sílica. A introdução do HPW só influenciou consideravelmente na condutividade da membrana a partir da quantidade de $3,93 \%$.

\section{Conclusões}

O trabalho apresenta relevância visto que o PVA é um polímero versátil que possibilita sua reticulação com uma ampla gama de materiais ${ }^{[25]}$. Além disso, atende a apelos ambientais uma vez que para o desenvolvimento do trabalho não foi utilizado nenhum solvente tóxico, a quantidade de partículas utilizadas foi reduzida, em comparação a trabalhos similares, e não houve necessidade de sulfonação do polímero. As membranas desenvolvidas mostraram características de adsorção e pervaporação melhores que as membranas de PVA puro indicando a ocorrência de reticulação e formação de membranas híbridas. O menor valor de condutividade alcançado, $0,02 \mathrm{mS} / \mathrm{cm}$, encontra-se na faixa de condutividade das membranas de PVA citadas na literatura ${ }^{[25]}$. O valor máximo de condutividade $(0,28 \mathrm{mS} /$ $\mathrm{cm})$ alcançado no trabalho foi através da membrana TH75. As membranas reticuladas com o precursor TEOS mostram ser uma boa alternativa para a reticulação de membranas de PVA visto que, no trabalho desenvolvido as membranas mostraram-se mais seletivas ao fluxo de solução de etanol. Entretanto, para aplicação como eletrólito de célula a combustível, ainda é necessário investigar outras formas de aumentar a condutividade sem prejudicar a estabilidade dimensional. A partir do conhecimento adquirido por este estudo, está sendo dada continuidade ao trabalho, seguindo esta metodologia, para identificar a quantidade de HPW adequada para aumentar a condutividade sem que ocorra o carreamento do mesmo devido a sua grande afinidade com a água.

\section{Agradecimentos}

Os autores agradecem a CAPES pelo suporte financeiro.

\section{Referências Bibliográficas}

1. Kim, Y. S. \& Pivovar, B. S. - "Polymer Electrolyte Membranes for Direct Methanol Fuel Cells", in: Advances in Fuel Cells, vol.1, cap.4, Tim Zhao, K.-D. Kreuer and Trung Van Nguyen (eds.), Elsevier Science, Kidlington (2007).

2. Thanganathan, U. \& Nogami, M. - J. Membr. Sci., 411, p.109 (2012). http://dx.doi.org/10.1016/j.memsci.2012.04.020.

3. Perles, C. E. - Polímeros., 18, p.281 (2008). http://dx.doi. org/10.1590/S0104-14282008000400005.

4. Zhong, G.; Liu, Z.; Li, T.; Cheng, H.; Yu, S.; Fu, R. \& Yang, Y. J. Membr. Sci., 428, p.212 (2013). http://dx.doi.org/10.1016/j. memsci.2012.10.026.

5. Aguiar, K. R.; Batalha, G. P.; Peixoto, M.; Ramos, A. \& Pezzin, S. H. - Polímeros., 22, p.453 (2012). http://dx.doi.org/10.1590/ S0104-14282012005000060.
6. Rhim, J.-W.; Park, H.; Lee, C.; Jun, J.; Kim, D. \& Lee, Y. - J. Membr. Sci., 238, p.143 (2004). http://dx.doi.org/10.1016/j. memsci.2004.03.030.

7. Martinelli, A.; Matic, A.; Jacobsson, P.; Borjesson, L.; Navarra, M.; Fernicola, A.; Panero, S. \& Scrosati, B. - Solid State Ion., 177, p.2431 (2006). http://dx.doi.org/10.1016/j. ssi.2006.01.035.

8. Shen, C.-C.; Joseph, J.; Lin, Y.-C.; Lin, S.-H.; Lin, C.-W. \& Hwang, B. J. - Desalination., 233, p.82 (2008). http://dx.doi. org/10.1016/j.desal.2007.09.029.

9. Zhu, G.; Wang, F.; Xu, K.; Gao, Q. \& Liu, Y. - Polímeros., 23, p.146 (2013). http://dx.doi.org/10.4322/polimeros.2013.076.

10. Kim, D. S.; Park, H.; Rhim, J. \& Lee, Y. - Solid State Ion., 176, p.117 (2005). http://dx.doi.org/10.1016/j.ssi.2004.07.011.

11. Huang, Y. F.; Chuang, L. C.; Kannan, A. M. \& Lin, C. W. - J. Power Sources., 186, p.22 (2009). http://dx.doi.org/10.1016/j. jpowsour.2008.09.072.

12. Guo, R.; Ma, X.; Hu, C. \& Jiang, Z. - Polymer (Guildf.)., 48, p.2939 (2007). http://dx.doi.org/10.1016/j. polymer.2007.03.035.

13. Wu, Y.; Wu, C.; Li, Y.; Xu, T. \& Fu, Y. - J. Membr. Sci., 350, p.322 (2010). http://dx.doi.org/10.1016/j.memsci.2010.01.007.

14. Nagarale, R. K.; Shin, W. \& Singh, P. K. - Polymer Chemistry., 1, p.388 (2010). http://dx.doi.org/10.1039/b9py00235a.

15. Ramos Filho, F. G. - "Membranas híbridas para uso em células a combustivel", Tese de Doutorado, Universidade Federal do Rio de Janeiro, Brasil (2009).

16. Guo, R.; Hu, C.; Pan, F.; Wu, H. \& Jiang, Z. - J. Membr. Sci., 281, p.454 (2006). http://dx.doi.org/10.1016/j. memsci.2006.04.015.

17. Bandyopadhyay, A.; De Sarkar, M. \& Bhowmick, A. K. - J. Mater. Sci., 41, p.5981 (2006). http://dx.doi.org/10.1007/ s10853-006-0254-x.

18. Nagarale, R. K.; Gohil, G. S.; Shahi, V. K. \& Rangarajan, R. - Macromolecules., 37, p.10023 (2004). http://dx.doi. org $/ 10.1021 / \mathrm{ma} 048404 \mathrm{p}$.

19. Kim, D. S.; Parka, H. B.; Rhimb, J. W. \& Lee, Y. M. - J. Membr. Sci., 240, p.37 (2004). http://dx.doi.org/10.1016/j. memsci.2004.04.010.

20. Gomes, A. S. \& Dutra Filho, J. C. - Int. J. Hydrogen Energ., 37, p.6246 (2011).

21. Xu, W.; Liu, C.; Xue, X.; Su, Y.; Lv, Y.; Xing, W. \& Lu, T. Solid State Ion., 171, p.121 (2004). http://dx.doi.org/10.1016/j. ssi.2004.04.009.

22. Mahreni, A.; Mohamad, A. B.; Kadhum, A. A. H.; Daud, W. R. W. \& Iyuke, S. E. - J. Membr. Sci., 327, p.32 (2009). http:// dx.doi.org/10.1016/j.memsci.2008.10.048.

23. Thiam, H. S.; Daud, W. R. W.; Kamarudin, S. K.; Mohammad, A. B.; Kadhum, A. A. H.; Loh, K. S. \& Majlan, E. H. - Int. J. Hydrogen Energy., 36, p.3187 (2011). http://dx.doi. org/10.1016/j.ijhydene.2010.11.062.

24. Li, L.; Xu, L. \& Wang, Y. - Mater. Lett., 57, p.1406 (2003). http://dx.doi.org/10.1016/S0167-577X(02)00998-9.

25. Maiti, J.; Kakati, N.; Lee, S. H.; Jee, S. H.; Viswanathan, B. \& Yoon, Y. S. - J. Power Sources., 216, p.48 (2012). http:// dx.doi.org/10.1016/j.jpowsour.2012.05.057.

Enviado: Out. 15, 2013

Reenviado: Mar. 11, 2014

Aceito: Maio 23, 2014 